\title{
Development of a fully nonlinear, coupled numerical model for assessment of floating tidal stream concepts
}

\author{
Brown, Scott Andrew
}

http://hdl.handle.net/10026.1/16650

10.1016/j.oceaneng.2020.108253

Ocean Engineering

Elsevier BV

All content in PEARL is protected by copyright law. Author manuscripts are made available in accordance with publisher policies. Please cite only the published version using the details provided on the item record or document. In the absence of an open licence (e.g. Creative Commons), permissions for further reuse of content should be sought from the publisher or author. 


\title{
Development of a fully nonlinear, coupled numerical model for assessment of floating tidal stream concepts
}

\author{
S.A. Brown ${ }^{\mathrm{a}, *}$, E.J. Ransley ${ }^{\mathrm{a}}$, S. Zheng ${ }^{\mathrm{a}}$, N. Xie ${ }^{\mathrm{a}, 1}$, B. Howey ${ }^{\mathrm{a}, 2}$, D.M. Greaves ${ }^{\mathrm{a}}$ \\ ${ }^{a}$ School of Engineering, Computing and Mathematics, University of Plymouth, Plymouth, United Kingdom
}

\begin{abstract}
Compared with existing seabed mounted systems, floating tidal concepts offer an increase in viable sites, along with the ability to harvest higher flow speeds near the free surface. However, such systems will be required to survive in highenergy wave-driven environments, and the performance and survivability of such systems is largely unknown. Current industry standards generally use a decoupled numerical approach to assess this problem, but this paper presents the development of a framework for an efficient numerical tool that is capable of predicting the fully nonlinear, coupled behaviour of floating tidal stream systems. Using the open-source CFD libraries of OpenFOAM as a basis, and a previously developed computationally efficient turbine model, the framework has been demonstrated using a generalised system. Verification against alternative codes based on linear potential theory shows good agreement when the floating structure is considered alone in linear waves. Systematic introduction of mooring forces and the turbine model demonstrates that it is necessary to consider all components of a floating tidal concept simultaneously, and that coupling between platform motion and fluid flow can substantially increase the cyclic amplitude of the turbine loading compared with a fixed device.
\end{abstract}

Keywords:

CFD, OpenFOAM, Actuator theory, HATT model, Marine Renewable Energy, Linear Potential Flow Theory

\section{Introduction}

In an effort to address the ever-present energy trilemma, the economic exploitation of renewable energy sources has become paramount for many of the World's governments (United Nations, 2015). For nations with sufficient resource, such as the UK (UK Government, 2009), research into the development of Offshore Renewable Energy (ORE) sources has become a priority area (UK Government, 2009) and due to its predictability, as well as its proximity to land, tidal stream energy has become an attractive option for a key role in the future energy mix (UK Government, 2009). Furthermore, the tidal stream industry has matured quickly by utilising technological developments made in existing industries, such as hydro and wind (particularly in the case of the Horizontal Axis Tidal Turbine (HATT)) (Fraenkel, 2002; Bahaj and Myers, 2003; Couch and Bryden, 2006; Batten et al., 2007; Ben Elghali et al., 2007; Greaves and Iglesias, 2018), and is almost cost-competitive with more established renewable energy sources (Greaves and Iglesias, 2018).

However, a major concern for the growing tidal stream industry is that there only appears to be a small num-

\footnotetext{
* Corresponding author

Email address: scott.brown@plymouth.ac.uk (S.A. Brown)

${ }^{1}$ Present Address: Department of Engineering, Saipem (UK) Ltd., Kingston upon Thames, United Kingdom

${ }^{2}$ Present Address: Plymouth Marine Laboratory, Plymouth, United Kingdom
}

ber of suitable sites for tidal stream projects (Greaves and Iglesias, 2018). This issue is compounded by an apparent convergence in the tidal stream technology towards seabed-mounted (or gravity-based devices) which impose limits on the range of water depths, as well as the nature of the bathymetry, at proposed sites (Greaves and Iglesias, 2018). In order to establish a supply chain for the industry, encourage investment and ensure economic viability, tidal stream technology needs to be developed to reduce the restrictions on possible development locations and increase the total potentially extractable resource (Couch and Bryden, 2006; Iglesias et al., 2012; Lewis et al., 2015).

In response to this, a number of floating tidal stream concepts have been proposed (Turnock et al., 2007; Røkke and Nilssen, 2013; Ransley et al., 2016; Shi et al., 2016; Policy and Innovation Group, 2017). Floating devices are less limited by water depth and bathymetry, greatly increasing the number of potential development sites; they also tend to be simpler and quicker to install, maintain and recover as they can often be towed to site, reducing the considerable expense associated with the use of specialist installation vessels (Lazakis et al., 2013). In addition to this, floating devices benefit from a turbine position towards the top of the water column, where the flow speed (and hence the resource) is greatest (Lewis et al., 2017; Greaves and Iglesias, 2018). This leads to a further increase in the potential resource when compared to projects involving seabed-mounted devices. 
Despite the considerable advantages of floating tidal stream concepts (over the more established seabed-mounted devices), they require a number of additional considerations to be made regarding their location at the freesurface. Not only do floating devices potentially have a greater impact on other stakeholders, e.g. navigational obstructions and aesthetic issues, but they are also exposed to free-surface effects and waves, raising concerns over both the power delivery and the survivability of the devices (Galloway et al., 2010; Nicholls-Lee, 2011; Faudot and Dahlhaug, 2012; Fernandez-Rodriguez et al., 2014; Olczak, 2016; Tatum et al., 2016). Presently, there is a distinct lack of understanding concerning the free-surface effects, wave loading and wave-induced motion of floating tidal stream devices and, therefore, considerable risk due to the uncertainties in the anticipated power output, cyclic loading of the turbine (Galloway et al., 2010; FernandezRodriguez et al., 2014; Olczak, 2016; Tatum et al., 2016) and predicted mooring line loads (Knappett et al., 2015; Weller et al., 2015). Further research is required, to improve the predicted behaviour, fatigue assessments and power delivery, before these devices will ever become commercially viable.

Due to the need to reduce risk, modelling (both physical and numerical) has become integral to the development of ORE devices ( $\mathrm{Li}$ and Yu, 2012; Adcock et al., 2015; Day et al., 2015; Borthwick, 2016). Numerical modelling, in particular, is being utilised consistently at low Technology Readiness Levels (TRLs) and has been shown to provide much of the quantitative description required for engineering design at a much lower cost when compared with physical modelling (Day et al., 2015). However, high-fidelity, coupled, modelling of floating structures is an emerging area of research in itself (Ransley et al., 2017a,b) and the case of a floating tidal stream device; with fully nonlinear hydrodynamics, wave-current interaction, coupled motion of a floating structure and mooring system, and (possibly multiple) submerged turbines; represent the very state of the art in numerical simulation.

Existing numerical codes are rarely capable of including all of these elements and, for those that are, the behaviour tends to be linearised and each of the elements treated separately, i.e. a 'decoupled' model. This creates considerable uncertainty in the power delivery and survivability predicted by these models (Galloway et al., 2010; Faudot and Dahlhaug, 2012; Fernandez-Rodriguez et al., 2014). A model which includes the contribution of all elements simultaneously as well as the fully nonlinear hydrodynamics, complex structural geometry and floating-body motion is therefore desirable when assessing the behaviour of these devices, the loads (in the mooring lines for example) and the power output from the turbine. Unfortunately, such a model, if available, would likely suffer from excessive CPU requirements making the use of such a tool prohibitive in routine design processes (Borg et al., 2014).

The aim of this work is, therefore, to develop an effi- cient numerical tool that is capable of predicting the fully nonlinear, coupled behaviour of floating tidal stream systems, including the motion of the structure, the loads in the mooring lines and the power output of the submerged turbine, and use it to assess the accuracy of more simplified methods.

This is the second article in a series that document the development, demonstration and application of the complete tool. In the first article a methodology is demonstrated, for a computationally efficient HATT model that can predict accurately the coupled forces on the turbine as well as remain stable throughout arbitrary motion through the computational mesh (Brown et al., 2020b). In this article the HATT model is coupled with the existing rigidbody motion solver from the open-source Computational Fluid Dynamics (CFD) libraries of OpenFOAM. This allows for simulation of floating structures with the additional, time-varying, thrust force from the turbine included in the equation of motion; the effect of which is demonstrated here through the idealised test case of a surfacepiercing, vertical cylinder connected to a submerged HATT with a single-point, taut mooring. The motion of the structure in waves is assessed through an incremental increase in complexity from a constrained case, excluding the turbine, to a freely floating, moored system with the turbine present. Results for the motion are compared against an analytical solution and those generated using Linear Potential Theory (LPT). The influence of the structure's motion on the power output of the turbine is then assessed and compared against a theoretical prediction. The coupled model is then used to simulate the device in a series of combined wave-current cases and the nature of the body motion and power output assessed with reference to more traditional linear and uncoupled methods.

\section{Methodology}

A new library, allowing for the representation of tidal turbines, has been designed and implemented in OpenFOAM v4.1 (Weller et al., 2017), an open-source toolbox aimed at solving continuum mechanics problems (including CFD). The software is written in $\mathrm{C}++$ and is based around the Object Orientated Programming (OOP) paradigm, offering a large collection of solvers and shared libraries. Consequently, the new turbine library is easily coupled with many of the existing solvers as previously demonstrated on single phase steady (simpleFOAM) and transient (pisoFOAM) solvers (Brown et al., 2020b). However, this study uses a transient two-phase approach and hence the turbine methodology is coupled with interFOAM (Rusche, 2002) which solves the incompressible ReynoldsAveraged Navier-Stokes (RANS) equations for two, incompressible, isothermal and immiscible fluids using the Finite Volume Method (FVM). Assuming laminar flow and neglecting surface tension, the incompressible (unsteady) 
RANS equations take the following form

$$
\begin{gathered}
\frac{\partial(\rho \mathbf{u})}{\partial t}+\nabla \cdot(\rho \mathbf{u u})=-\nabla p+\nabla^{2}(\mu \mathbf{u})+\rho \mathbf{g}+\mathbf{T}, \\
\nabla \cdot \mathbf{u}=0,
\end{gathered}
$$

where $p$ is the pressure; $\mathbf{u}=(u, v, w)$ is the fluid velocity; $\rho$ is the fluid density; $\mu$ is the dynamic viscosity; $g$ is acceleration due to gravity; and $\mathbf{T}$ is the momentum sink due to the presence of the turbine. The two-phase solver interFOAM uses the Volume Of Fluid (VOF) method to track the interface between the two phases, which are considered to be a single continuous fluid with variable density and viscosity according to

$$
\begin{aligned}
& \rho=\alpha_{1} \rho_{0}+\left(1-\alpha_{1}\right) \rho_{1}, \\
& \mu=\alpha_{1} \mu_{0}+\left(1-\alpha_{1}\right) \mu_{1},
\end{aligned}
$$

where subscripts 0 and 1 represent air and water, respectively. The phase fraction, $\alpha_{1}$, is a non-dimensional parameter that represents the phase averaged volume fraction in each cell, and ranges from zero (representing a cell containing only air) to one (representing a cell containing only water). The interface can then be found from values of $\alpha_{1}$ such that $0<\alpha_{1}<1$.

A similar 'blending approach' is used to treat the velocities of the two phases

$$
\mathbf{u}=\alpha_{1} \mathbf{u}_{0}+\left(1-\alpha_{1}\right) \mathbf{u}_{1},
$$

and the RANS equations are then solved using these 'blended' values, with the VOF advection equation,

$$
\frac{\partial \alpha_{1}}{\partial t}+\nabla \cdot\left(\mathbf{u} \alpha_{1}\right)=0,
$$

solved separately (based on the velocities obtained) to determine the new location of the interface between the two fluid phases.

\subsection{Turbine Model}

Although highly-detailed flow structure around a tidal turbine can be obtained in CFD via a blade-resolved approach, this methodology's large computational requirements generally limit its use to studies focusing on specific details relating to an isolated turbine, such as performance changes due to turbulence (Lloyd et al., 2014); surface-gravity waves (Tatum et al., 2016); and the interaction with a diffuser (Song et al., 2019). In the present application where the turbine only forms a single component of the entire system, the computational expense of the blade-resolved approach is infeasibly large if all parts of the system are to be modelled simultaneously in a coupled approach. Hence, in this work, a more computationally efficient approach has been adopted using a "weighted body force implementation' which, at each time step, identifies and applies weights to a region of the computational domain (representing the turbine), determines the local velocity at the turbine position and adds an additional, equal and opposite force, $T$ (based on the thrust on the turbine), to the momentum equations (equation 1) (Brown et al., 2020b). A summary of the key steps of the method is given in this section with the reader referred to Brown et al. (2020b) for full details.

The method relies on calculating the free stream velocity, $u_{\infty}$, (and hence thrust, $T$, on the turbine) based on the local velocity in the region of the turbine, $u_{t}$. This is achieved by reverse engineering actuator theory (Burton et al., 2001),

$$
u_{\infty}=\frac{u_{t}}{(1-a)}, \quad T=\frac{1}{2} \rho C_{t} A u_{\infty}^{2},
$$

where $a$ is the axial induction factor, $A$ is the swept area of the turbine and $C_{t}=4 a(1-a)$ is the turbine thrust coefficient (Brown et al., 2020b). The local velocity, $u_{t}$, is considered to be the axial component of the weighted average of the instantaneous velocity (relative to the platform's motion) in each of the mesh cells,

$$
\mathbf{u}_{a v}=\left(\frac{1}{V} \sum_{i=1}^{N} \mathbf{u}_{i} V_{i} W_{i}\right)-\mathbf{v}_{h u b},
$$

where $N$ is the total number of cells in the turbine region; $V_{i}$ is the volume in the $i^{\text {th }}$ cell; $\mathbf{v}_{h u b}$ is the velocity at the turbine hub; $V$ is the total weighted volume of the turbine region

$$
V=\sum_{i=1}^{N} V_{i} W_{i} .
$$

The weighting of the $i^{\text {th }}$ cell $\left(W_{i}\right)$, is determined based on the relative proximity of that cell to the centre of the turbine,

$$
\mathbf{d} \mathbf{x}=\mathbf{x}_{c e l l}-\mathbf{x}_{h u b}=(d x, d y, d z), \quad \mathbf{r}=(0, d y, d z),
$$

and follows a Gaussian distribution in the turbine region,

$$
W= \begin{cases}\frac{1}{\sigma \sqrt{2 \pi}} \exp \left(-\frac{d x^{2}}{2 \sigma^{2}}\right) & \text { if }|d x| \leq n \sigma \text { and }|\mathbf{r}| \leq R \\ 0 & \text { otherwise }\end{cases}
$$

where $\sigma$ is the Gaussian root mean square width (Brown et al., 2020b) and $n$ is a user-defined coefficient (set by default to 2 according to the sensitivity analysis in Brown et al. (2020b)).

\subsection{Coupling with Rigid Body Solver}

In order to simulate floating tidal energy concepts, the methodology outlined in Section 2.1 and Brown et al. (2020b) has been coupled with OpenFOAM's sixDoFRigidBodyMotion solver which allows for rigid body motion of a structure in Six Degrees Of Freedom (6DOF) using the existing dynamic mesh motion libraries. The sixDoFRigidBodyMotion solver itself has already 
been thoroughly validated for other floating renewable energy devices (Ransley et al., 2017a; Brown et al., 2020a; Ransley et al., 2020), but the present coupling introduces additional complexity into the turbine method since the turbine is now able to move in six degrees of freedom. In Brown et al. (2020b) the turbine methodology was shown to be robust when passing through a mesh at a prescribed velocity (in translation and rotation) but, here, the instantaneous location of the turbine cells will be arbitrary and must be determined using the orientation and position of the platform. Therefore, the cells corresponding to the turbine region are now selected by rotating the coordinate system based on the orientation matrix of the body, $\mathbf{Q}_{b o d y}$, i.e.

$$
\mathbf{d} \mathbf{x}^{\prime}=\mathbf{Q}_{b o d y}^{T} \cdot\left(\mathbf{Q}_{0}^{T} \cdot \mathbf{d} \mathbf{x}\right),
$$

where $\mathbf{Q}_{0}$ is the initial orientation of the turbine calculated according to

$$
\mathbf{Q}_{0}(\alpha, \beta, \gamma)=\mathbf{R}_{x}\left(\frac{\alpha}{2}\right) \mathbf{R}_{y}\left(\frac{\beta}{2}\right) \mathbf{R}_{z}(\gamma) \mathbf{R}_{y}\left(\frac{\beta}{2}\right) \mathbf{R}_{x}\left(\frac{\alpha}{2}\right)
$$

where $\mathbf{R}_{x}, \mathbf{R}_{y}, \mathbf{R}_{z}$ are matrices defining a rotation about the global $x, y$ and $z$ axes respectively. These are defined as

$$
\begin{aligned}
\mathbf{R}_{x} & =\left(\begin{array}{ccc}
1 & 0 & 0 \\
0 & \cos (\alpha) & -\sin (\alpha) \\
0 & \sin (\alpha) & \cos (\alpha)
\end{array}\right), \\
\mathbf{R}_{y} & =\left(\begin{array}{ccc}
\cos (\beta) & 0 & \sin (\beta) \\
0 & 1 & 0 \\
-\sin (\beta) & 0 & \cos (\beta)
\end{array}\right), \\
\mathbf{R}_{z} & =\left(\begin{array}{ccc}
\cos (\gamma) & -\sin (\gamma) & 0 \\
\sin (\gamma) & \cos (\gamma) & 0 \\
0 & 0 & 1
\end{array}\right),
\end{aligned}
$$

where $\alpha, \beta$ and $\gamma$ are the angles of roll, pitch and yaw respectively.

The turbine model calculation then proceeds as described in Section 2.1 (with $\mathbf{d x}$ replaced by $\mathbf{d x}^{\prime}$ ). The velocity at the centre of the turbine, $\mathbf{v}_{h u b}$ is determined based on the linear $(\mathbf{v})$ and angular $(\boldsymbol{\omega})$ velocities of the system, assuming that the structure is rigid,

$$
\mathbf{v}_{h u b}=\left(\boldsymbol{\omega} \times\left(\mathbf{x}_{h u b}-\mathbf{x}_{C o M}\right)+\mathbf{v}\right),
$$

where $\mathbf{x}_{C o M}$ is the position of the centre of mass. The thrust force on the turbine, $T$ (equation 7 ), is then included in the equation of motion (equation 1) of the body as an additional force acting at a position coincident with that of the turbine hub, $\mathbf{x}_{h u b}$. The 6 DOF solver updates both the acceleration, a, and torque, $\tau$, on the body, at each time step,

$$
\begin{gathered}
\mathbf{a}=\mathbf{a}_{\text {fluid }}+\mathbf{a}_{\text {turbine }}+\mathbf{a}_{\text {mooring }}, \\
\tau=\tau_{\text {fluid }}+\tau_{\text {turbine }}+\tau_{\text {mooring }}
\end{gathered}
$$

where

$$
\begin{gathered}
\mathbf{a}_{\text {turbine }}=\frac{T \mathbf{x}_{\text {axis }}}{m}, \\
\tau_{\text {turbine }}=\mathbf{Q}_{\text {body }}^{T} \cdot\left(\tau_{t}+\left(\mathbf{x}_{\text {hub }}-\mathbf{x}_{C o M}\right) \times T \mathbf{x}_{\text {axis }}\right),
\end{gathered}
$$

and

$$
\mathbf{x}_{a x i s}=\mathbf{Q}_{b o d y}^{T} \cdot\left(\mathbf{Q}^{T} \cdot \hat{\mathbf{x}}\right),
$$

is the axial direction of the turbine in the global coordinate system. Here $m$ is the mass of the body; $\tau_{t}$ is the torque imparted by the turbine at the hub position, $\mathbf{x}_{h u b} ; \mathbf{a}_{\text {fluid }}$ and $\tau_{\text {fluid }}$ are the contributions from the fluid loading to the acceleration and torque imposed on the body respectively, and; $\mathbf{a}_{\text {mooring }}$ and $\tau_{\text {mooring }}$ are the contributions from the mooring system as described in Section 2.3.

In the turbine model presented here, the torque imparted by the turbine, $\tau_{t}$, is set to zero, i.e. the scenario is akin to freely rotating blades with no power extraction via the generator (pure thrust). In future work, turbine models will be created that include the torque due to energy extraction via the turbine; these are easily implemented due to the OOP structure of the developments discussed in this work.

\subsection{Moorings}

Station-keeping is a key aspect in the design of floating tidal energy concepts and, typically, this is achieved using a system of moorings and anchors. In this study, simple moorings are considered for demonstration purposes but the implementation is again easily adapted to accommodate more complicated mooring systems via the OOP paradigm.

The moorings are expressed as an additional force (a 'restraint') in the equation of motion of the body, similar to the approach used for the turbine described above. The mooring force from each mooring line, $\mathbf{F}_{\text {mooring, }}$, is calculated using a slightly adapted version of Hooke's law (for a linear spring) and is applied at a predefined attachment point that moves rigidly with the body, $\mathbf{x}_{\text {mooring }}$, in the direction of a vector joining $\mathbf{x}_{\text {mooring }}$ to a predefined anchor position, $\mathbf{x}_{\text {anchor }}$. The acceleration due to a single mooring is then given by:

$$
\mathbf{a}_{\text {mooring }}=\frac{\mathbf{F}_{\text {mooring }}}{m},
$$

where

$$
\mathbf{F}_{\text {mooring }}= \begin{cases}-k\left(|\mathbf{l}|-l_{0}\right) \hat{\mathbf{l}} & \text { if }|\mathbf{l}|>l_{0} \\ (0,0,0) & \text { if }|\mathbf{l}| \leq l_{0},\end{cases}
$$

$k$ is the mooring stiffness, $l_{0}$ is the rest length of the mooring and

$$
\mathbf{l}=\mathbf{x}_{\text {mooring }}-\mathbf{x}_{\text {anchor }},
$$

is a vector, inline with the mooring force, with a magnitude equal to the current length of the mooring. 
Similarly, as in the case of the turbine (equation 21), the torque on the body due to a mooring line is given by

$$
\tau_{\text {mooring }}=\mathbf{Q}_{\text {body }}^{T} \cdot\left(\left(\mathbf{x}_{\text {mooring }}-\mathbf{x}_{C o M}\right) \times \mathbf{F}_{\text {mooring }}\right) .
$$

In this work, the moorings are assumed to be massless and have been designed so that there is no reaction force due to compression, i.e. when the mooring is slack (equation 24).

\subsection{The General Case}

In the most general case, a floating tidal stream concept may consist of a buoyant platform with any number of mooring lines and multiple submerged turbines. The developments discussed here have been designed in such a way that this general case is easily implemented. For a device with $n$ mooring lines and $j$ turbines, equations 18 and 19 simply become

$$
\begin{gathered}
\mathbf{a}=\mathbf{a}_{\text {fluid }}+\sum_{j} \mathbf{a}_{\text {turbine }, j}+\sum_{n} \mathbf{a}_{\text {mooring }, n} \\
\tau=\tau_{\text {fluid }}+\sum_{j} \tau_{\text {turbine }, j}+\sum_{n} \tau_{\text {mooring }, n}
\end{gathered}
$$

\section{Test Case}

To demonstrate the capabilities of the framework developed here, an idealised test case is considered, using a vertical, surface-piercing, cylindrical structure with radius $r=6 \mathrm{~m}$, length $l=3 \mathrm{~m}$, and draft $d=1.5 \mathrm{~m}$ for the platform. This geometry is chosen to allow for comparison with analytical and benchmarking solutions; the dimensions are chosen to give a waterplane area, mass and draft similar to those of an existing floating tidal stream concept (Ransley et al., 2016).

Using the additional toolbox waves2Foam (Jacobsen et al., 2012), regular waves of height $H=0.3 \mathrm{~m}$ are generated, using stream function wave theory (Dean, 1965; Fenton, 1988), in a water depth of $h=20 \mathrm{~m}$. A range of wave periods, from $T=3 \mathrm{~s}$ to $T=5.5 \mathrm{~s}$, are chosen to sample the frequency space around the analytical natural frequency of the structure, $f_{0}$ (see Section 3.1). Consequently, the waves considered here are all deep water waves of either Stokes $1^{\text {st }}$ or $2^{\text {nd }}$ order; it is believed that this constraint allows for reasonable comparison with linear models, i.e. those typically used in engineering design practices.

The CFD numerical domain has a width ( $y$-axis) and height (z-axis) of $30 \mathrm{~m}$ and $32 \mathrm{~m}$ respectively. To absorb reflected waves, Relaxation Zones (RZs) are positioned at both the outlet and inlet boundaries of the numerical domain (Jacobsen et al., 2012). The length of the numerical domain has, therefore, been parametrically designed such that the lengths (in the direction of wave propagation $(x$-axis $))$ of the inlet RZ, working section and outlet RZ are 0.5 wavelengths $(L), 1.5 L$ and $1.5 L$ respectively.

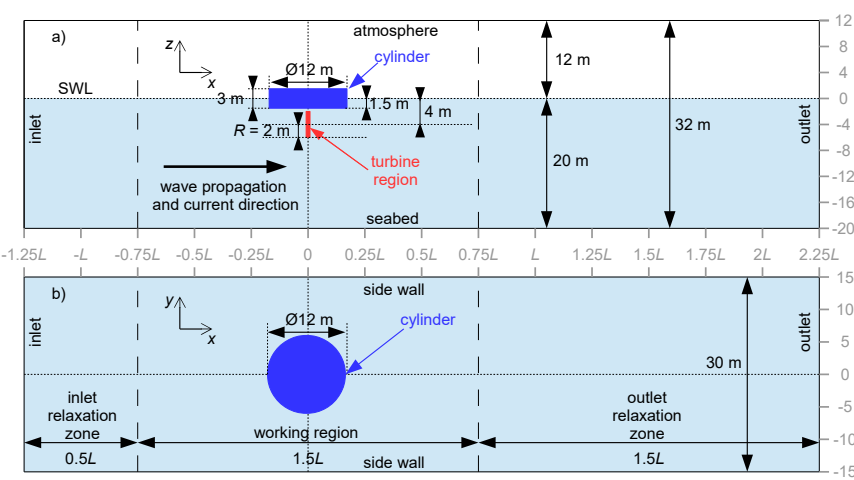

Figure 1: Numerical domain used for the simulations of the generic coupled system in the $x-z(\mathrm{a})$ and $x-y(\mathrm{~b})$ planes.

The outlet RZ is purposely longer than normally required for wave-only conditions, i.e. for $0.1 \%$ reflection (Jacobsen et al., 2012), as it has been found that in combined wavecurrent conditions a longer RZ is necessary to achieve the same level of absorption as that in wave-only cases (Ransley et al., 2016). The centre of the cylinder is situated at the origin of the domain, which is located at the Still Water Level (SWL), $1.25 L$ (in the $x$-direction) from the inlet boundary (Figure 1).

The numerical domain is discretised initially with cubic cells (side length $\Delta x=0.4 \mathrm{~m}$; Aspect Ratio of 1). The free surface region $(-0.2 \mathrm{~m} \leq z \leq 0.3 \mathrm{~m})$ and mesh around the structure are then refined by two octree levels (Greaves and Borthwick, 1999), so that the discretisation is $0.1 \mathrm{~m}$ (Figure 2), giving 3 cells over the wave height. For the steepest wave considered $(T=3 \mathrm{~s})$ this is anticipated to give a Root Mean Squared (RMS) tolerance of $2 \%$, but for the majority of the wave periods considered the tolerance is expected to be $1 \%$ or less due to reduced steepness (Ransley, 2015). Additionally, there is a $5 \mathrm{~m}^{3}$ refinement region of two octree levels $(\Delta x=0.1 \mathrm{~m})$ with its centre coincident with the centre of the turbine region (see Section 4 for turbine details). Since the turbine is not present in all of the simulations discussed in this section, the mesh does not necessarily need to be refined in this region. However, it was considered necessary to keep the mesh consistent throughout so that any effects arising from mesh dependency were minimised.

The side walls of the domain and the seabed (Figure 1) are treated as solid walls and have non-slip boundary conditions applied for velocity. The cylinder is also considered to be a solid object and uses a modified non-slip condition that accounts for the movement of the boundary. The inlet and outlet velocities are set to the desired velocity profile, i.e. combined wave and current at the inlet and current only at the outlet. The velocity values at the atmosphere boundary are calculated according to the near-cell pressure with the pressure on the boundary set to the total 


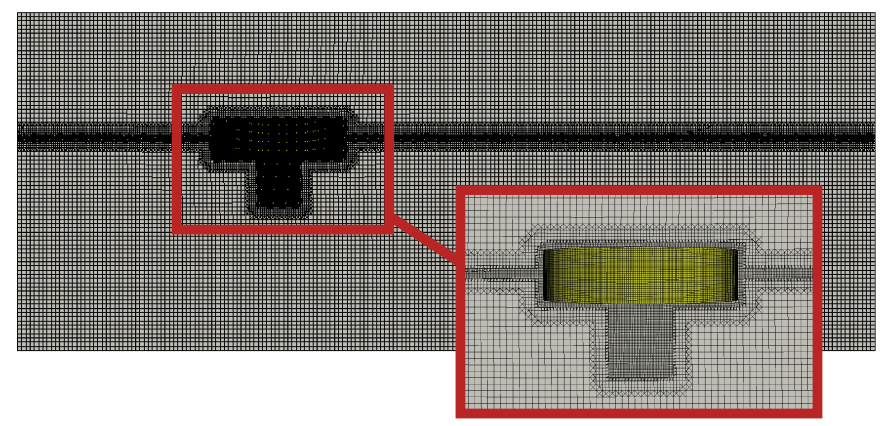

Figure 2: Visualisation of the mesh structure used in this work.

pressure, defined as

$$
p=p_{0}+\frac{1}{2} \rho|\mathbf{u}|^{2},
$$

where $p_{0}$ is a user-defined reference value set to zero in this work since the solver uses the difference between total and hydrostatic pressure.

A mesh independence study was conducted to quantify the anticipated numerical uncertainty following the method of Eça and Hoekstra (2014), which has been successfully demonstrated previously for similar floating applications (Eskilsson et al., 2017; Brown et al., 2020a). Four meshes were considered, each using the same refinement structure as described above but with a different initial discretisation $(\Delta x=0.8 \mathrm{~m}, 0.6 \mathrm{~m}, 0.4 \mathrm{~m}$ and $0.2 \mathrm{~m})$. The $\mathrm{T}=4 \mathrm{~s}$ wave-only case has been considered, and it is assumed that the numerical uncertainty will be similar for the remaining cases. The results of this mesh independence study indicate that the chosen mesh leads to a numerical uncertainty of $5 \%, 4 \%$ and $1.5 \%$ for the surge, heave and pitch degrees of freedom, respectively.

Each simulation is run for 12 wave periods and the Response Amplitude Operators (RAOs) are calculated using the 6 periods after a stable periodic solution is found. The heave and pitch RAOs are calculated by identifying the zero up-crossings in the time series, calculating the amplitudes of the motion between subsequent zero-crossings, and taking the mean of the amplitudes. In cases where the surge motion is unconstrained, the surge generally includes a significant drift component superposed onto the periodic response, hence, additional processing is required: a moving average is removed from the data using a window width of 1 wave period; the remaining data is then periodic and the same procedure as that used for the heave and pitch RAOs is utilised. Throughout this section, 95\% confidence intervals, calculated using 2 standard deviations of the set of amplitudes, are also presented.

\subsection{Industry Standard Verification}

For verification purposes and to provide a baseline solution, this section presents a comparison of the RAOs obtained for the cylinder only, i.e. no moorings or turbine, between the present, fully nonlinear model and the current industry standard for predicting responses to wave excitation. The most commonly used approaches are based upon Linear Potential Theory (LPT), and predict the wave induced forces on structures in frequency-space through calculation of the hydrodynamic coefficients (added mass, $a$, radiation damping, $b$ and excitation force, $\left.F_{e}\right)$. LPT assumes that the flow is both inviscid and irrotational to allow the problem to be formulated in terms of the velocity potential, and must satisfy boundary conditions on the body, seabed, free surface and far field.

This problem can be solved using multiple numerical approaches including Eigen function expansion (Zheng and Zhang, 2015) and Boundary Element Methods (BEMs) based on the Free Surface Green function (Lee and Newman, 2004), or Rankine source panel method (Xie and Vassalos, 2012). The Eigen function expansion method uses separation of variables to expand the velocity potentials using Bessel functions (Siddorn and Eatock Taylor, 2008; Zheng and Zhang, 2015), which satisfy the free surface, sea bottom and far field conditions. The unknown coefficients of the expanded velocity potentials are determined using the body boundary condition and continuity of velocity and pressure at the domain interface. By using a form of Green's Theorem (Lee and Newman, 2004), the Free Surface Green function method satisfies all of the conditions except the one on the body, and therefore only the source distribution on the mean wetted hull surface must be considered in order to obtain the diffraction and radiation velocity potentials. The Rankine panel method uses Rankine source (Xie and Vassalos, 2012) as the primary solution of the Laplace equation, and does not satisfy any of the boundary conditions for the velocity potentials. Therefore, all of the domain boundaries need to be discretised and sources distributed on them (Xie and Vassalos, 2007, 2012).

In theory, all of these three methods should produce the same hydrodynamic coefficients and, hence, wave excitation forces for a particular floating structure. Therefore, the user must determine the suitability of each method for a particular problem based on the relative advantages and disadvantages. The Eigen function expansion method is capable of analysing thousands of frequencies in the order of minutes because it does not require discretisation of the boundaries, making it the most computationally efficient method considered here, although it is only suitable for bodies of specified regular shape e.g. circular cylinders (Garrett, 1971; Zheng and Zhang, 2015). The Free Surface Green function method can be applied more generally for 3-D bodies, and since the source only needs to be distributed on the wetted hull surface, the resultant equation is relatively small (Faltinsen, 1993), making it a commonly used technique in available panel method codes (e.g. ANSYS Aqwa (ANSYS, Inc., 2019), NEMOH (L'École Centrale De Nantes, 2019)). However, the Free Surface Green function method is not suitable for nonlinear free surface flow problems. Rankine source based methods have the flexibility to alter the free surface boundary condition in 

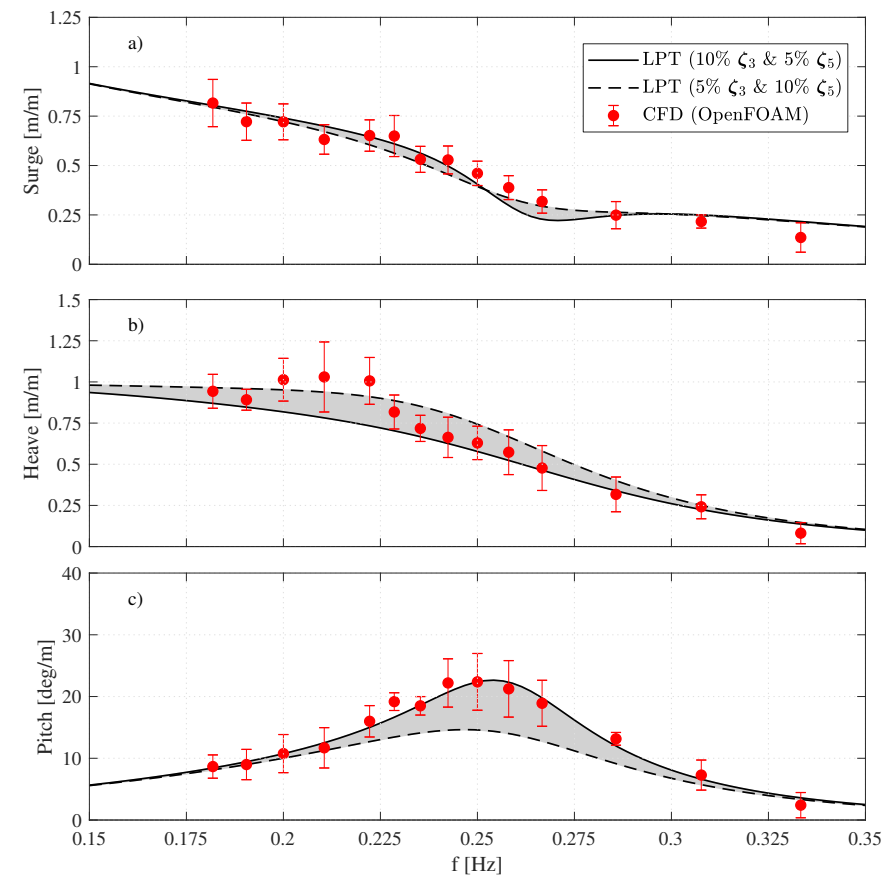

Figure 3: Comparison of the CFD $(\bullet)$ predictions (with $95 \%$ confidence interval) of surge (a), heave (b), and pitch (c) RAOs with LPT solutions using additional damping: $\boldsymbol{\zeta}_{3}=10 \%$ and $\boldsymbol{\zeta}_{5}=5 \%(-)$; $\boldsymbol{\zeta}_{3}=5 \%$ and $\boldsymbol{\zeta}_{5}=10 \%(---)$; and anticipated region for CFD results (shaded area).

order to consider higher order problems, even fully nonlinear free surface boundary conditions, viscous effects (resonance problem) and different sea bottom topologies. However, due to the need to distribute the source on all flow boundaries, the resultant equations are relatively larger (compared with Free Surface Green function methods) and hence require more computational effort to resolve.

Despite this, all of the methods are still substantially cheaper computationally than CFD, and generally perform acceptably for idealised wave-only conditions, making them a useful design tool for simplified systems. However, they tend to exclude the contributions of other parts of the system (e.g. moorings and installed turbines) or treat them as decoupled. The comparison presented in this section is solely intended to verify that the CFD results are agreeable with industry standards in simplified conditions, ensuring that the results can be used as a reliable benchmark to determine any complications due to other components of the floating tidal system (Sections 4 and 5). Therefore, LPT solutions are presented that have been obtained using an in-house Eigen function expansion code (Zheng and Zhang, 2015) (without prior knowledge of the CFD results), and have been verified independently using Free Surface Green function (ANSYS Aqwa (ANSYS, Inc., 2019), NEMOH (L'École Centrale De Nantes, 2019)) and Rankine source (in-house (Xie and Vassalos, 2007, 2012)) based BEMs for further confidence in the solution.

Figure 3 presents a comparison of the CFD (•) predictions (with $95 \%$ confidence interval) of surge (a), heave (b), and pitch (c) RAOs with the LPT solution. Additional damping (representing viscous effects), denoted $\mathbf{B}^{\nu}$ here, is usually required in these models to avoid unphysically large responses at the resonance frequencies, and is estimated based on the critical damping

$$
\mathbf{B}_{j, j}^{\nu}=2 \boldsymbol{\zeta}_{j} \sqrt{\left(\mathbf{M}_{j, j}+\mathcal{M}_{j, j}\right) \mathbf{K}_{j, j}}
$$

where the subscript $j$ represents the $j^{\text {th }}$ element of the inertial $(\mathbf{M})$, added mass $(\boldsymbol{M})$ and hydrostatic stiffness $(\mathbf{K})$ matrices. The parameter $\boldsymbol{\zeta}$ is a user-defined value representing an estimated percentage in each Degree Of Freedom (DOF). Based on experience (Xie and Vassalos, 2012) and analysis of previous structures (Himeno, 1981; Kawahara et al., 2009), it is thought (without prior knowledge of the CFD results) that $5-10 \%$ is a suitable estimation for both heave $\left(\boldsymbol{\zeta}_{3}\right)$, and pitch $\left(\boldsymbol{\zeta}_{5}\right)$, with none in the other DOFs. Furthermore, following sensitivity analysis of the LPT solutions, it is found that heave is independent of the additional damping due to pitch, and vice versa in this case, whilst surge is also dependent on the additional pitch. Therefore, two solutions are presented representing the upper and lower bounds: the low heave $\left(\boldsymbol{\zeta}_{3}=5 \%\right)$, high pitch $\left(\boldsymbol{\zeta}_{5}=10 \%\right)$ case $(---)$; and the high heave $\left(\boldsymbol{\zeta}_{3}=10 \%\right)$, low pitch $\left(\boldsymbol{\zeta}_{5}=5 \%\right)$ case $(-)$; with the expectation that the CFD results will lie in between the two (shaded area).

The CFD results are agreeable with the LPT solution for all DOFs, and generally lie within the shaded region as hypothesised. Figure 3 a shows that there are minor discrepancies in the surge RAO: for instance at $f \approx 0.27 \mathrm{~Hz}$, there is a minimum in the high heave, low pitch $\left(10 \% \zeta_{3} \&\right.$ $\left.5 \% \boldsymbol{\zeta}_{5}\right)$ solution which are not observed in the CFD; but overall, it is considered to be captured acceptably. The CFD predictions of the heave RAO (Figure 3b), generally agree better with the high heave $\left(10 \% \boldsymbol{\zeta}_{3}\right)$, rather than low heave $\left(5 \% \boldsymbol{\zeta}_{3}\right)$ case, implying that $10 \%$ is a good estimation for viscous damping in this case. However, the responses to frequencies near the peak $(0.2 \mathrm{~Hz} \leq f \leq 0.225 \mathrm{~Hz})$ are over-estimated using this higher damping, although it is noted that there is lower confidence (higher variability) in the results at these frequencies. This is likely due to radiated waves being reflected from the side walls of the domain (where there is no absorption), which could be reduced by increasing the width of the domain or applying lateral wave absorption methods. In pitch (Figure 3c), the CFD solutions are in good agreement with LPT, and generally follow the low pitch $\left(5 \% \boldsymbol{\zeta}_{5}\right)$ very well, indicating that an additional viscous damping of $5 \%$ is suitable.

Overall, the present CFD results agree well with the LPT model for the small amplitude linear waves considered here, verifying that the CFD solution can be reliably used as the base solution when introducing additional components of the system, as discussed in Sections 4 and 5 . 

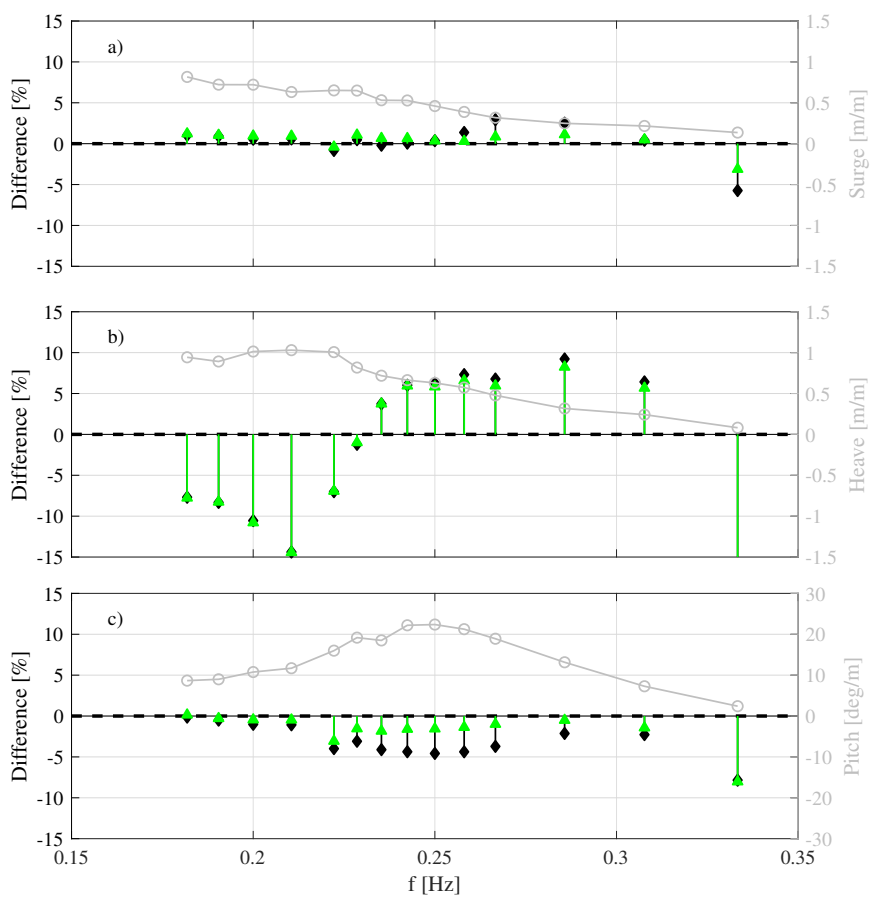

Figure 4: Change [\%] in a) surge, b) heave, and c) pitch RAO predictions relative to the CFD results presented in Section 3.1, through the inclusion of the mooring $(\Delta)$, and the turbine and mooring combined $(\diamond)$. For reference, the base solutions (CFD results from Section 3.1) are also presented $(\circ)$.

\section{Coupled Modelling in Waves}

In Section 3.1, it was verified that the present model captures the RAOs of a floating surface-piercing cylinder in agreement with other popular methods used by the offshore industry. However, the real benefit of the present model is its ability to accommodate additional forces arising from moorings and the submerged turbine that make up a complete floating tidal energy concept. The coupled nature of these additional elements is rarely included when modelling these devices and so, in this section, the effect of evaluating the entire coupled system is compared against the CFD results from Section 3.1 to demonstrate the important discrepancies in present design practices.

For this demonstration, a single mooring line is used for station keeping with a rest length, $l_{0}$, of $18.2 \mathrm{~m}$ and stiffness $k=41875 \mathrm{~N} \mathrm{~m}^{-2}$. The stiffness of the mooring has been chosen by scaling up an existing mooring which was used in physical experiments in the COAST laboratory at the University of Plymouth (Hann et al., 2015). When at rest, the mooring attachment is at $(0,0,-1.5)$, in the centre of the bottom face of the cylinder, the mooring is vertical and the anchor position is on the seabed at $(0,0,-20)$.

The turbine parameters are set to $R=2, C_{t}=0.9$, $\sigma=0.15$ and $N=2$ giving an anticipated error in the predicted free-stream velocity of only $1 \%$ (Brown et al., $2020 \mathrm{~b})$. The centre of the turbine is located centrally, $2.5 \mathrm{~m}$ below the bottom of the structure $(z=-4 \mathrm{~m})$ (Fig- ure 1). The turbine support structure is neglected for simplicity and, to allow for direct comparison, the turbine is assumed to be massless so that the mass of the system and the rest position are the same with and without the turbine included.

Figure 4 presents the differences, relative to the structure only, wave-only CFD results presented in Section 3.1, in the predicted surge (a), heave (b), and pitch (c) RAOs when the model includes: the mooring only $(\Delta)$, or; both the mooring and turbine $(\bullet)$. For reference, the structure only CFD results obtained in Section 3.1, used as the base solution, are also presented (०).

Including only the mooring does not substantially affect the surge (Figure 4a) or pitch (Figure 4c) RAOs, with differences generally $2 \%$ or less compared to the CFD results in Section 3.1. This is thought to be due to the use of a relatively compliant single point mooring, rather than a four-point mooring design which would constrain both the surge and pitch motion more effectively. On the other hand, the mooring does significantly alter the heave RAO (Figure 4b), with differences of up to $15 \%$. Including the mooring damps the heave motion of the system around the unmoored peak frequency and increases the response at higher frequencies implying a shift in the peak response towards those high frequencies.

With both the single point mooring and turbine included $(\bullet)$, the surge (Figure $4 \mathrm{a}$ ) and heave (Figure 4b) RAOs do not differ substantially from the moored-only cases. The lack of observed differences in the surge response is a little surprising, but is thought to be a consequence of the relatively low flow velocities under small waves, resulting in a low thrust on the turbine. It is anticipated that in more realistic flows, i.e. larger waves as well as currents, the thrust exerted on the turbine would have a much larger effect on the surge RAO (see Section 5). Despite this, even with the low thrust observed in these small wave-only cases, the pitch RAO is reduced markedly around the peak frequency of the CFD results from Section 3.1. This demonstrates that having the turbine thrust coupled to the motion of the cylinder adds additional damping to the pitch of the system and indicates that, even in relatively benign cases, inclusion of the turbine can significantly change the behaviour of the device. This further justifies the need for a tool which can consider the fully-coupled nature of floating tidal systems.

\subsection{Motion-Thrust Coupling}

The present numerical model shows that the combination of waves, moorings and turbine substantially change the devices motion, and that it is essential to use a coupled model to capture these changes. However, developers are also likely to be interested in the platform motion's influence on the turbine, since any changes in thrust due to the motion will potentially alter the turbine fatigue rate and complicate power delivery. Therefore, this section analyses the platform's motion-thrust coupling by comparing turbine thrust in moored and fixed conditions. Since 

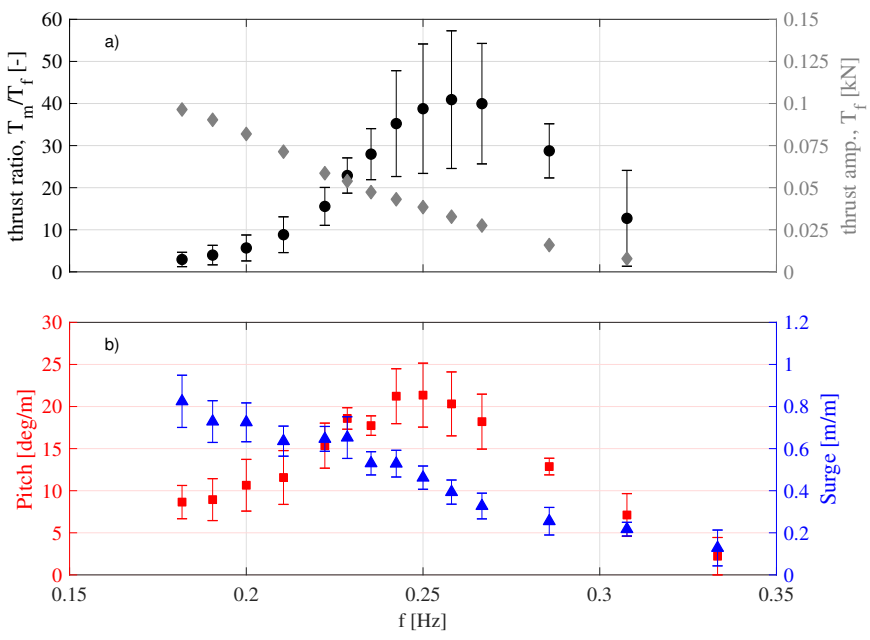

Figure 5: Thrust ratio, $T_{m} / T_{f}(\bullet)$ and fixed amplitude $T_{f}(\bullet)$ as a function of frequency (a); Pitch ( $\mathbf{\square})$ and surge $(\mathbf{\Delta})$ RAOs with mooring and turbine in wave-only conditions (b). The error bars represent the $95 \%$ confidence interval based on two standard deviations of the wave periods used for sampling.

the effects of wave-induced motion on turbines is a crucial uncertainty in the development of floating tidal systems, wave-only conditions are used for the analysis in this section to avoid complications caused by currents. Therefore, the turbine thrust amplitude has been obtained by simulating the device in a fixed (equilibrium) position using the range of wave frequencies considered in Section 3.1.

The fixed turbine thrust amplitude $\left(T_{f}\right)$ decreases with increasing wave frequency ( $\checkmark$ in Figure $5 \mathrm{a}$ ) due to the reduced horizontal velocities in these conditions. Interestingly, the turbine thrust amplitudes in moored conditions $\left(T_{m}\right)$ are substantially increased in comparison to the loads experienced in fixed conditions: Figure 5 a presents the ratio $(\bullet)$ of moored and fixed amplitudes $\left(T_{m} / T_{f}\right)$ and shows that the increase is frequency dependent and ranges from $3-40$ times larger in the wave conditions simulated here. This considerable increase is thought to be related to the motion of the device: considering the pitch RAO, obtained using the same wave periods as $T_{m}$, and presented in Figure $5 \mathrm{~b}(\mathbf{\square})$, it follows a similar trend as the relative thrust increase, indicating that the two parameters are related. The values presented here are likely to be exaggerated due to the large motion relative to the low flow velocities in wave-only conditions, but an increase due to the motion is still probable in more realistic conditions due to the turbine acting like a large lever-arm in pitch, making it a critical consideration for developers. The surge was also expected to be a factor in the load cycling, but the RAO $(\boldsymbol{\Delta})$ does not follow any obvious pattern with the thrust ratio, although it does display a similar trend to the thrust on the fixed turbine. This latter observation is likely due to both parameters primarily being functions of drag, and consequently the square of the horizontal flow velocity (equation 7 ).

The error bars in Figure 5 (95\% confidence interval) in-
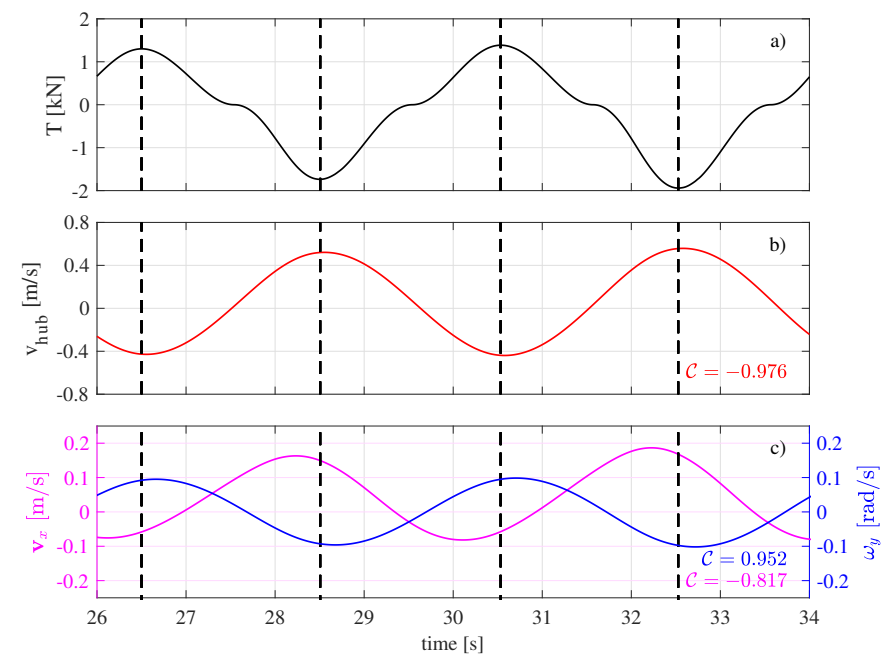

Figure 6: Time series of $T(\mathrm{a}) ; v_{h u b}(\mathrm{~b}) ; \mathbf{v}_{\mathbf{x}}(-)$ and $\boldsymbol{\omega}_{y}(-)$ $(\mathrm{c})$; in $f=0.25 \mathrm{~Hz}$ waves. The cross-correlation $(\mathcal{C})$ between each variable and the thrust is shown in the corresponding colour.

dicate that there is substantially more variability in thrust amplitude than the RAOs, thought to be due to high sensitivity to changes in pitch due to the lever-arm effect. The variability in pitch and surge is likely caused by reflections in the numerical tank from a number of sources: the outlet relaxation zone, side walls or interaction of radiated waves with the incident wave, which can be mitigated through increased domain size.

The influence of both the surge and pitch parameters is likely to be a function of their phases relative to the wave velocity, and hence geometry of the device and mooring design. Figure 6 presents time series of the turbine thrust (a), axial velocity at the centre of the turbine, $v_{h u b}$, (b) the horizontal velocity, $\mathbf{v}_{\mathbf{x}}(-)$, and angular velocity about the y axis, $\boldsymbol{\omega}_{y}$ (-), of the cylinder (c) in the $f=0.25 \mathrm{~Hz}$ case. The axial velocity has been obtained using equations (17) and (22), i.e.

$$
v_{h u b}=\mathbf{x}_{a x i s} \cdot \mathbf{v}_{h u b} .
$$

The times of maximum (or minimum) turbine thrust are shown in each plot $(---)$, and these occur close to times of minimum (or maximum) axial velocity (Figure 6b, ) indicating a strong negative correlation (cross-correlation coefficient, $\mathcal{C}=-0.976$ ). The negative relationship is due to the turbine observing a positive relative velocity when $v_{h u b}$ is negative (equation 8 ). There is a very slight discrepancy between the peaks in thrust and $v_{h u b}$, which is likely due to the influence of the flow velocity and its phase relationship with the platforms motion. The angular velocity about the y-axis (Figure 6c, - ) has a similarly strong correlation with thrust $(\mathcal{C}=0.952$, positive due to the definition of the axis), providing further evidence that the pitch is the dominant factor in this problem. However, the peaks do occur slightly later than $v_{h u b}$ due to the horizontal linear velocity of the system (Figure $6 \mathrm{c}$, $-)$, which is less considerable in the present application 
but does influence the results slightly.

This section highlights the necessity to consider the whole system as a single coupled problem, since thrust on the turbine both influences and is influenced by the motion of the platform, which in turn is strongly two-way coupled with the mooring dynamics. Although the relative increase in thrust amplitude due to motion is expected to be less significant in more realistic wave-currents conditions due to increased flow velocities (and hence a smaller offset between motion and flow), it should still be a key consideration for any developer due to the implications on power delivery and fatigue. The present numerical framework could be a key design tool especially in systems with complex geometries, or flow conditions, where the motion/flow phase relationship could be substantially different to that observed here.

\section{Coupled Modelling in Wave-Currents}

In Section 4 it is shown that even in wave-only conditions, i.e. at times of slack water in a tidal cycle, it is necessary to consider the entire coupled system to predict the precise behaviour of a floating tidal stream device. However throughout the majority of the time of deployment, the device is subject to combined wave-current conditions. Therefore, in this section, the new framework has been used to model the behaviour of the device in a range of wave-current conditions using a subset of wave frequencies from Section 4.

\subsection{Pre-processing and Initialisation}

In the combined wave-current cases, a series of preprocessing and initialisation steps are undertaken to: account for the addition drag force on the device (due to the currents); ensure a high quality mesh is retained throughout the simulations, and; minimise the time taken to reach a stable equilibrium state.

First, the anchor point of the mooring is repositioned at $\left(-x_{e q, u}, 0,-20\right)$ where $x_{e q, u}$ is the theoretical equilibrium position for each current speed, $u$. The equilibrium position is determined by considering the horizontal force balance between the mooring tension (equation 24) and the thrust forces on the cylinder, $T_{c y l}$, and the turbine, $T$ (equation 7). As a first approximation (assuming the thrust on the cylinder is dominated by drag forces),

$$
T_{c y l, u}=\frac{1}{2} \rho C_{D} r l u^{2},
$$

where $C_{D}$ is the drag coefficient and is set to 1 in this case (Morison et al., 1950). Considering the displacement in surge only, the equilibrium position, $x_{e q, u}$, can be approximated by satisfying

$$
T_{c y l, u}+T_{u}=-k\left(1-\frac{l_{0}}{\sqrt{x_{e q, u}^{2}+x_{0}^{2}+y_{0}^{2}+z_{0}^{2}}}\right) x_{e q, u}
$$
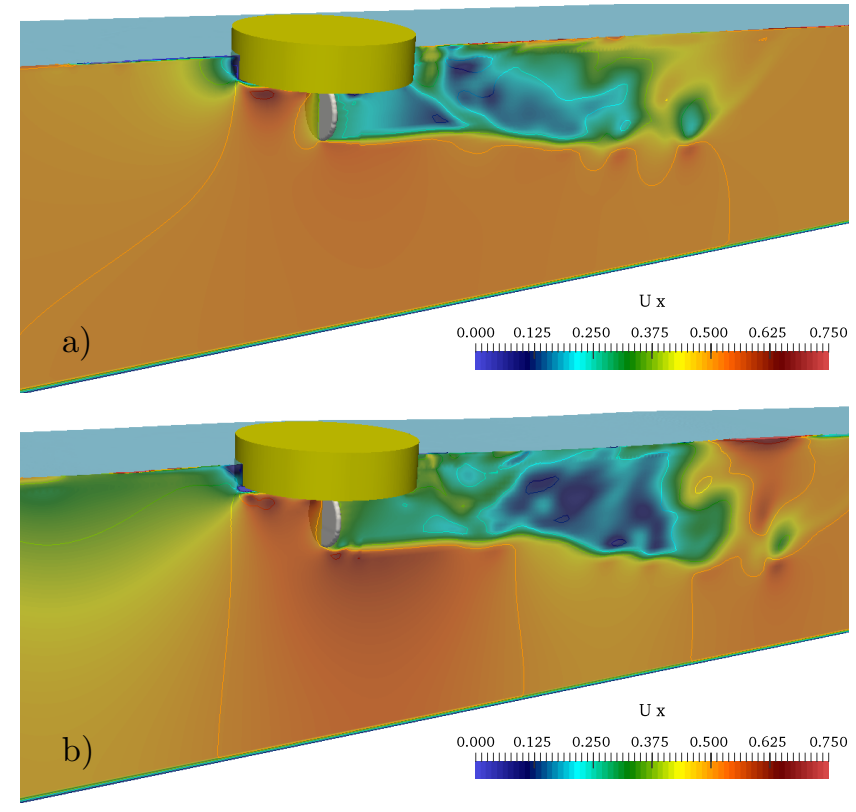

Figure 7: Snapshots of the case setup including the cylinder, turbine region and horizontal velocity field for the $u=0.5, f=0.2 \mathrm{~Hz}$ case: Initial (current-only) conditions achieved through the initialisation processes described in Section 5.1 (a); and the numerical solution after $30 \mathrm{~s}$ of wave-current conditions (b).

where $\left(x_{0}, y_{0}, z_{0}\right)=(0,0,18.5)$ are the initial streamwise, transverse and vertical lengths of the mooring respectively.

In the wave-current cases, the flow field variables are initialised in two stages: 1) the model is run for $60 \mathrm{~s}$, in current only conditions, with the cylinder fixed; this ensures the turbine thrust and velocity field reach a pseudoequilibrium state before the cylinder is allowed to move. 2) The cylinder is released and a further $30 \mathrm{~s}$ is simulated to allow the system to reach the actual (rather than theoretical) equilibrium position (Figure $7 \mathrm{a}$ ).

Interestingly, it was found that the thrust on the turbine consistently converged to a value $10-20 \%$ larger than the theoretical solution for the prescribed inlet condition. This could potentially be due to the use of a transient solver but, since the model has been shown to be converged to around $1 \%$ on a similar mesh in an unblocked steady case (Brown et al., 2020b), this increase in thrust is likely due to a combination of blockage effects caused by the presence of the free surface (Myers and Bahaj, 2010; Kolekar and Banerjee, 2015), the relatively shallow water depth (Myers and Bahaj, 2010; Nishino and Willden, 2012; Consul et al., 2013) and the proximity of the cylinder to the turbine region (see increased flow speed between turbine and cylinder in Figure 7a). This is an interesting observation as an increase in thrust is effectively an increase in power generated by the turbine and so could be of significant value to device developers. The increased thrust causes the surge equilibrium location to increase from the theoretical prediction (equation 33) making the second initialisation stage crucial. However, running $90 \mathrm{~s}$ of CFD simulation for initialisation purposes is not accept- 

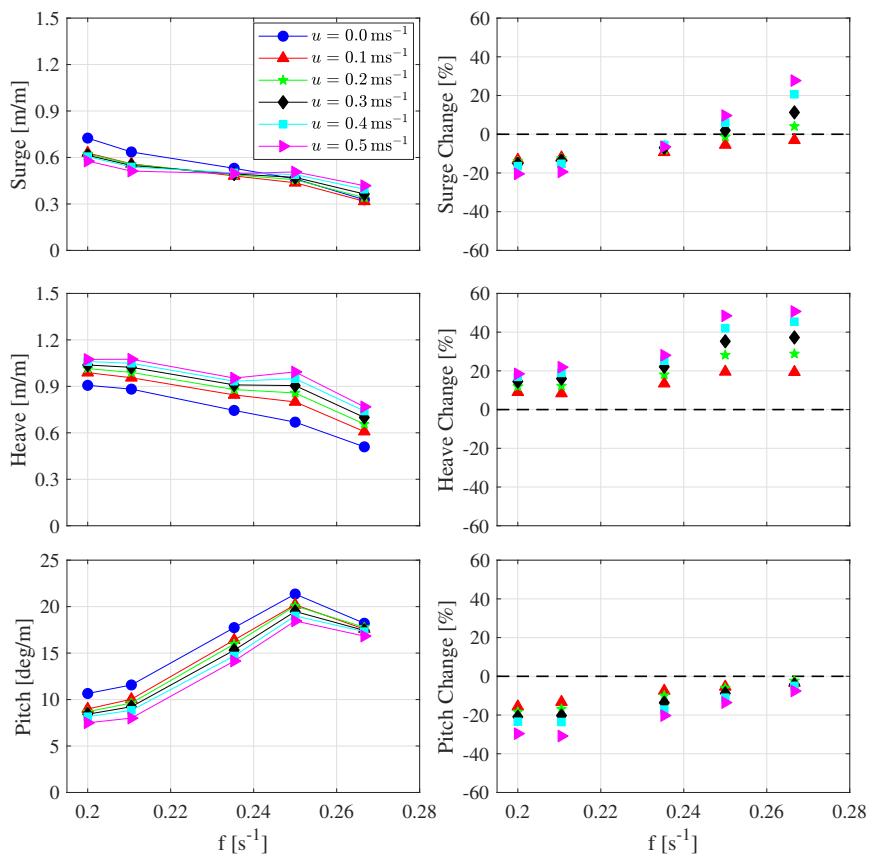

Figure 8: Surge (a), heave (c) and pitch (e) RAOs curves of the moored device with turbine, for a range of current speeds: $0.0(\bullet)$, $0.1(\Delta), 0.2(\star), 0.3(\bullet), 0.4(\square)$ and $0.5 \mathrm{~ms}^{-1}(\bullet)$. Also shown is the relative change of each DOF from the wave-only solution (b,d,f).

able in routine design practices and future efforts should be made to streamline the process. This could potentially be achieved by providing an improved initial guess for the velocity field, either using an analytical or less computationally expensive numerical approach such as a steadystate simulation to provide the initial conditions.

\subsection{Results}

Using the initialised conditions (described in Section 5.1), the structure is assessed in a range of wave-current conditions (Figure $7 \mathrm{~b}$ ). A subset of the wave frequencies used in Section $4(0.2 \mathrm{~Hz} \leq f \leq 0.27 \mathrm{~Hz}$, chosen to include both heave and pitch peak frequency) are superposed on a range of current speeds $\left(0.1 \mathrm{~ms}^{-1} \leq u \leq 0.5 \mathrm{~ms}^{-1}\right)$, using the method described in Section 5.1. Note that the inlet Reynolds numbers $\left(2 e^{6} \leq R e \leq 1 e^{7}\right)$ for the presented flow speeds imply turbulent flow. However, since the case presented in this work is purely designed to demonstrate the need for coupled modelling, laminar flow is still assumed to allow comparison with the wave-only cases without the added complication of turbulence.

Figure 8 presents the surge (a), heave (c) and pitch (e) RAO curves of the moored device with turbine, for a range of current speeds: $0.0(\bullet), 0.1(\boldsymbol{\Delta}), 0.2(\star), 0.3$ $(\diamond), 0.4(\square)$ and $0.5 \mathrm{~ms}^{-1}(\triangleright)$. Also shown is the relative change of each DOF from the wave only solution (b,d,f). The heave response (Figure $8 \mathrm{~b}, \mathrm{~d}$ ) can be seen to increase substantially (up to 50\%) with current speed, for all of the wave frequencies. Such an increase in heave response is slightly unexpected since wave height is anticipated to re- duce slightly in the presence of a following current. Therefore, it is hypothesised that the increase may be due to the offset in surge caused by the combination of turbine and current: i.e. since the mooring force no longer acts solely in the vertical direction the structure may have more freedom to move in heave.

The pitch response (Figure 8e,f) shows the opposite trend to the heave; as the current speed increases, the pitch response reduces (by up to 30\%), indicating that the pitch damping due to the turbine and mooring combination (observed originally in Figure 4c) increases with current speed. Furthermore, the lower frequencies show a much larger relative change than the higher frequencies, indicating that there may be a shift in the peak pitch frequency.

Unlike the other two degrees of freedom, the change in surge response (Figure 8a,b) due to currents appears to be heavily influenced by the wave frequency. The lower frequencies show a decrease in surge amplitude, whereas the higher frequencies generally increase the response, with both increasing with current speed (up to $\pm 30 \%$ ). The middle frequency considered $(f=0.235 \mathrm{~Hz})$ is not sensitive to the current speed since the model predicts a similar decrease $(\approx 10 \%)$ for all current cases. These observations indicate that the surge peak frequency is being changed by a combination of the current, turbine and mooring, and that $f=0.235$ is close to an inflection point in Figure $8 \mathrm{~b}$.

\section{Conclusions}

A novel and efficient computational framework for modelling complete floating tidal systems is presented. The method utilises OpenFOAM's two-phase 6DOF rigid body solver, algebraic restraints for the moorings and a bodyforce type technique to include the influence of a submerged turbine. The framework of the coupled model is demonstrated via a simplified concept comprising a cylindrical barge, single point linear mooring and an actuator disc turbine. An incremental approach is used to determine the effect of each component in the system. The predicted response of the structure (without mooring or turbine) in linear waves is verified against industry standard codes based on LPT, generally showing good agreement. Addition of the mooring and turbine show changes of around 5\%, in linear waves, relative to the unrestrained data. The changes in the wave-only cases are too small to justify the additional computational expense of the present model (compared to alternatives, such as LPT), but in the wave-current conditions more substantial changes (up to $\pm 50 \%$ ) are observed in each DOF (surge, pitch and heave). When considering the motion-turbine coupling, the motion is shown to increase substantially the amplitude of the thrust on the turbine (up to $4000 \%$ ), in wave-only conditions. This is attributed to a combination of the pitch of the device and the distance of the turbine from the centre of rotation. Although this effect is amplified in wave-only conditions, due to the high magnitudes of motion relative 
to flow velocity, an increase is still likely in more realistic conditions. Hence, it is vital that the full motion-turbine coupling of the system is considered in order to fully understand the potential implications in terms of fatigue and power delivery.

The changes caused by considering the full tidal system in combined wave-current conditions using a fully nonlinear, coupled manner are substantial, especially when considering that the simulations were run using small amplitude linear waves and significantly lower flow speeds than would be observed at a realistic tidal test site. These sites will consistently have to deal with combinations of high flow speeds and large amplitude non-linear waves, potentially interacting with the system from any direction, as well as being able to survive extreme events. It is essential to be able to evaluate the performance, motion and survivability of a full floating tidal system in these conditions before deploying a prototype, especially when considering the substantial changes experienced in the relatively benign conditions presented in this work. The numerical framework presented here is capable of evaluating all of these parameters as well as providing high-volumes of additional information regarding the flow dynamics (Figure 7) which could be used to provide design decisions regarding arrays or assess the environmental impacts. Although the presented numerical model has the potential to improve confidence during the development stage of floating tidal devices in the future, the work presented here is purely intended to demonstrate the framework and therefore focuses on an idealised problem with a relatively low complexity turbine model. This provides the foundation for future research on the behaviour of floating tidal systems in more realistic conditions but the model will first require validation against scaled laboratory data. Following the validation stage, it will provide a method to assess scaling effects that cannot be easily evaluated without building a full-scale prototype. Additionally, the OOP nature of the code allows the potential for more sophisticated turbine and mooring models to be implemented, allowing for more detailed hydrodynamic analysis, and could be extended to similar applications such as floating wind turbines.

\section{Acknowledgements}

The authors acknowledge that, this work was funded as part of Innovate UK Projects 102217 and 103499 through the Energy Catalyst programme, with additional support from the Engineering and Physical Sciences Research Council (EPSRC) via the Partnership for Research in Marine Renewable Energy [EP/P026109/1] and the Supergen ORE Hub [EP/S000747/1]. The code presented here is available through the Collaborative Computational Project in Wave Structure Interaction (CCP-WSI) [EP/M022382/1], which aims to bring together the community of researchers, data, code and expertise within the area of wave structure interaction (WSI) and has the objective of building an opensource, community-driven numerical wave tank (NWT) fa- cility and capacity for high quality research in an area of high importance (https://www.ccp-wsi.ac.uk).

\section{References}

Adcock, T., Draper, S., Nishino, T., 2015. Tidal power generation - a review of hydrodynamic modelling. Proceedings of the Institution of Mechanical Engineers, Part A: Journal of Power and Energy 229, 755-771.

ANSYS, Inc., 2019. ANSYS Aqwa. website. https://www.ansys . com/products/structures/ansys-aqwa accessed 1st March 2019.

Bahaj, A., Myers, L., 2003. Fundamentals applicable to the utilisation of marine current turbines for energy production. Renewable Energy 28, 2205-2211.

Batten, W., Bahaj, A., Molland, A., Chaplin, J., 2007. Experimentally validated numerical method for the hydrodynamic design of horizontal axis tidal turbines. Ocean Engineering 34, 1013-1020.

Ben Elghali, S., Benbouzid, M., Charpentier, J., 2007. Marine tidal current electric power generation technology: state of the art and current status., in: IEEE Internation Electric \& Drives Conference, pp. 1407-1412.

Borg, M., Shires, A., Collu, M., 2014. Offshore floating vertical axis wind turbines, dynamics modelling state of the art. part I: aerodynamics. Renewable and Sustainable Energy Reviews 39, 1214-1225.

Borthwick, A., 2016. Marine renewable energy seascape. Engineering $2,69-78$

Brown, S., Musiedlak, P.H., Ransley, E., Greaves, D., 2020a. Quantifying the predictive capability of OpenFOAM 5.0: Focused wave impacts with floating bodies. International Journal of Offshore and Polar Engineering 30, 20-27.

Brown, S., Ransley, E., Greaves, D., 2020b. Developing a coupled turbine thrust methodology for floating tidal stream concepts: Verification under prescribed motion. Renewable Energy 147, 529540 .

Burton, T., Jenkins, N., Sharpe, D., Bossanyi, E., 2001. Wind Energy Handbook. 2 ed., John Wiley \& Sons, Ltd.

Consul, C., Willden, R., McIntosh, S., 2013. Blockage effects on the hydrodynamic performance of a marine cross-flow turbine. Philosophical Transactions of the Royal Society A 371, 16.

Couch, S., Bryden, I., 2006. Tidal current energy extraction: hydrodynamic resource characteristics. Proceedings of the Institution of Mechanical Engineers, Part M: Journal of Engineering for the Maritime Environment 220, 185-194.

Day, A., Babarit, A., Fontaine, A., He, Y.P., Kraskowski, M., Murai, M., Penesis, I., Salvatore, F., Shin, H.K., 2015. Hydrodynamic modelling of marine renewable energy devices: A state of the art review. Ocean Engineering 108, 46-69.

Dean, R.G., 1965. Stream function representation of nonlinear water waves. Journal of Geophysical Research 70, 4561-4572.

Eça, L., Hoekstra, M., 2014. A procedure for the estimation of the numerical uncertainty of CFD calculations based on grid refinement studies. Journal of Computational Physics 262, 104-130.

Eskilsson, C., Palm, J., Bergdahl, L., 2017. On numerical uncertainty of VOF-RANS simulations of wave energy converters through $\mathrm{v}$ $\& \mathrm{v}$ technique, in: Proceedings of the 12th European Wave and Tidal Energy Conference, Cork, Ireland. p. 18.

Faltinsen, O.M., 1993. Sea Loads on Ships and Offshore Structures. 1st ed., Cambridge University Press.

Faudot, C., Dahlhaug, O., 2012. Prediction of wave loads on tidal turbine blades. Energy Procedia 20, 116-133.

Fenton, J.D., 1988. The numerical solution of steady water wave problems. Computers \& Geosciences 14, 357-368.

Fernandez-Rodriguez, E., Stallard, T., Stansby, P., 2014. Experimental study of extreme thrust on a tidal stream rotor due to turbulent flow and with opposing waves. Journal of Fluids and Structures 51, 354-361.

Fraenkel, P., 2002. Power from marine currents. Proceedings of the Institution of Mechanical Engineers, Part A: Journal of Power and Energy 216, 1-14. 
Galloway, P., Myers, L., Bahaj, A., 2010. Studies of a scale tidal turbine in close proximity to waves, in: Proceedings of the 3rd International Conference on Ocean Energy, Bilbao, Spain. pp. 16 .

Garrett, C., 1971. Wave forces on a circular dock. Journal of Fluid Mechanics 46, 129-139.

Greaves, D., Borthwick, A., 1999. Hierarchical tree-based finite element mesh generation. Int. J. Numer. Meth. Eng. 45, 447-471.

Greaves, D., Iglesias, G., 2018. Wave and Tidal Energy. John Wiley \& Sons Ltd.

Hann, M., Greaves, D., Raby, A., 2015. Snatch loading of a single taut moored floating wave energy converter due to focussed wave groups. Ocean Engineering 96, 258-271.

Himeno, Y., 1981. Prediction of Ship Roll Damping - State of the Art. Technical Report 239. Department of Naval Architecture \& Marine Engineering, University of Michigan.

Iglesias, G., Sánchez, M., Carbello, R., Fernández, 2012. The TSE index - a new tool for selecting tidal stream sites in depth-limited regions. Renewable Energy 48, 350-357.

Jacobsen, N.G., Fuhrman, D.R., Fredsøe, J., 2012. A wave generation toolbox for the open-source CFD library: OpenFOAM ${ }^{\circledR}$. International Journal for Numerical Methods in Fluids 70, 10731088 .

Kawahara, Y., Maekawa, K., Ikeda, Y., 2009. Simple prediction formula of roll damping of conventional cargo ships on the basis of ikeda's method and its limitation, in: Proceedings of the 10th International Conference on Stability of Ships and Ocean Vehicles, Sat. Petersburg. pp. 387-398.

Knappett, J., Brown, M., Aldaikh, H., Patra, S., O'Laughlin, C., Chow, S., Gaudin, C., Lieng, J., 2015. A review of anchor technology for floating renewable energy devices and key design considerations., in: Proceedings of the 3rd International Symposium on Frontiers in Offshore Geotechnics, Oslo, Norway. pp. 887-892.

Kolekar, N., Banerjee, A., 2015. Performance characterization and placement of a marine hydrokinetic turbine in a tidal channel under boundary proximity and blockage effects. Applied Energy 148, $121-133$.

Lazakis, I., Turan, O., Rosendahl, T., 2013. Modeling of vessel and equipment cost for the maintenance activities of an offshore tidal energy array, in: PRADS: The 12th Internation Symposium on Practical Design of Ships and Other Floating Structures, Changwon City, Korea. pp. 1-9.

L'École Centrale De Nantes, 2019. Nemoh-toolbox. website. https://lheea.ec-nantes.fr/logiciels-et-brevets/ nemoh-toolbox-192933.kjsp accessed 1st March 2019.

Lee, C.H., Newman, J., 2004. Computation of wave effects using the panel method, in: Numerical Modeling in Fluid-Structure Interaction, WIT Press. p. 41

Lewis, M., Neill, S., Robins, P., Hashemi, M., 2015. Resource assessment for future generations of tidal-stream energy arrays. Energy $83,403-415$.

Lewis, M., Neill, S., Robins, P., Hashemi, M., Ward, S., 2017. Characteristics of the velocity profile at tidal-stream energy sites. Renewable Energy 114, 258-272.

Li, Y., Yu, Y.H., 2012. A synthesis of numerical methods for modeling wave energy converter-point absorbers. Renew. Energ. 37, 285-298.

Lloyd, T., Turnock, S., Humphrey, V., 2014. Assessing the influence of inflow turbulence on noise and performance of a tidal turbine using large eddy simulations. Renewable Energy 71, 742-754.

Morison, J., O'Brien, M.P., Johnson, J.W., Schaaf, S.A., 1950. The forces exerted by surface waves on piles. Journal of Petroleum Technology 2, 149-154.

Myers, L., Bahaj, A., 2010. Experimental analysis of the flow field around horizontal axis tidal turbines by use of scale mesh disk rotor simulators. Ocean Engineering 37, 218-227.

Nicholls-Lee, R.F., 2011. Adaptive composite blades for horizontal axis tidal turbines. Ph.D. thesis. University of Southampton.

Nishino, T., Willden, R., 2012. Effects of 3-D channel blockage and turbulent wake mixing on the limit of power extraction by tidal turbines. International Journal of Heat and Fluid Flow 37, 123-
135

Olczak, A., 2016. The influence of waves on tidal stream turbine arrays. Ph.D. thesis. University of Manchester.

Policy and Innovation Group, 2017. UK Ocean Energy Review 2017. Technical Report. University of Edinburgh.

Ransley, E., 2015. Survivability of Wave Energy Converter and Mooring Coupled System using CFD. Ph.D. thesis. University of Plymouth.

Ransley, E., Brown, S., Greaves, D., Hindley, S., Weston, P., Guerrini, E., Starzmann, R., 2016. Coupled RANS-VOF modelling of floating tidal stream concepts., in: Proceedings of the 4th Marine Energy Technology Symposium (METS), Washington, D.C., USA. p. 5 .

Ransley, E., Greaves, D., Raby, A., Simmonds, D., Hann, M., 2017a. Survivability of wave energy converters using CFD. Renewable Energy 109, 235-247.

Ransley, E., Greaves, D., Raby, A., Simmonds, D., Jakobsen, M. Kramer, M., 2017b. RANS-VOF modelling of the wavestar point absorber. Renewable Energy 109, 49-65.

Ransley, E., Yan, S., Brown, S., Hann, M., Graham, D., Windt, C., Schmitt, P., Davidson, J., Ringwood, J., Musiedlak, P.H., Wang, J., Wang, J., Ma, Q., Xie, Z., Zhang, N., Zheng, X., Giorgi, G., Chen, H., Lin, Z., Qian, L., Ma, Z., Bai, W., Chen, Q., Zang, J., Ding, H., Cheng, L., Zheng, J., Gu, H., Gong, X., Liu, Z., Zhuang, Y., Wan, D., Bingham, H., Greaves, D., 2020. A blind comparative study of focused wave interactions with floating structures (CCPWSI Blind Test Series 3). International Journal of Offshore and Polar Engineering 30, 1-10.

Røkke, A., Nilssen, R., 2013. Marine current turbines and generator preference. a technology review., in: Proceedings of the International Conference on Renewable Energies and Power Quality, Bilbao, Spain. p. 6

Rusche, H., 2002. Computational fluid dynamics of dispersed twophase flows at high phase fractions. Ph.D. thesis. Imperial College of Science, Technology \& Medicine.

Shi, W., Atlar, M., Rosli, R., Aktas, B., Norman, R., 2016. Cavitation observations and noise measurements of horizontal axis tidal turbines with biomimetic blade leading-edge designs. Ocean Engineering 121, 143-155.

Siddorn, P., Eatock Taylor, R., 2008. Diffraction and independent radiation by an array of floating cylinders. Ocean Engineering 35, 1289-1303.

Song, K., Wang, W.Q., Yan, Y., 2019. Numerical and experimental analysis of a diffuser-augmented micro-hydro turbine. Ocean Engineering 171, 590-602.

Tatum, S., Allmark, M., Frost, C., O’Doherty, D., Mason-Jones, A., O'Doherty, T., 2016. CFD modelling of a tidal stream turbine subjected to profiled flow and surface gravity waves. International Journal of Marine Energy 15, 156-174.

Turnock, S., Muller, G., Nicholls-Lee, R., Denchfield, S., Hindley, S., Shelmerdine, R., Stevens, S., 2007. Development of a floating tidal energy system suitable for use in shallow water, in: Proceedings of the 7th European Wave and Tidal Energy Conference, Porto, Portugal. p. 9

UK Government, 2009. National Renewable Energy Action Plan for the United Kingdom - Article 4 of the Renewable Energy Directive 2009/28/EC. Technical Report. UK Government.

United Nations, 2015. Paris Agreement. Technical Report. United Nations Treaty Collection.

Weller, H., Greenshields, C., Bainbridge, W., Janssens, M., Santos, B., 2017. OpenFOAM 4.1. URL: https://openfoam.org/ version/4-1/.

Weller, S., Johanning, L., Davies, P., Banfield, S., 2015. Synthetic mooring ropes for marine renewable energy applications. Renewable Energy 83, 1268-1278.

Xie, N., Vassalos, D., 2007. Performance analysis of 3D hydrofoil under free surface. Ocean Engineering 34, 1257-1264.

Xie, N., Vassalos, D., 2012. Evaluation of the m-term and 3D ship motion in waves. Journal of Ship Mechanics 16, 971-979.

Zheng, S., Zhang, Y., 2015. Wave diffraction from a truncated cylinder in front of a vertical wall. Ocean Engineering 104, 329-343. 